\title{
Regional Cerebral Blood Flow in Normotensive and Spontaneously Hypertensive Rats
}

\author{
Jun-ichiro Choki, Takenori Yamaguchi, Yasuyuki \\ Morotomi, Yo Takeya, and Teruo Omae
}

Bilateral ligation of the common carotid arteries in spontaneously hypertensive rats (SHR) is known to cause a much more increase in anaerobic metabolites in the brain than in normotensive Wistar rats (Fujishima $M$ et al: Stroke $6: 62,1975$ ), and to cause a marked decrease in reactivity of cortical microvasculature to vasodilating agents (Yamaguchi $\mathrm{T}$ et al: J Jap Coll Angiol, in press). These findings suggest that bilateral carotid ligation may cause more severe ischemic damages to the brain in SHR. Actual values of ccrcbral blood flow, however, were not measured in these experiments.

The present experiment was designed to compare the regional cerebral blood flow (rCBF) values in SHR with those in normotensive Wistar rats (NTR), before and after the ligation of bilateral common carotid arteries.

Thirteen NTR and 15 SHR ( 7 to 12 months old) were anesthetized lightly with amobarbital $(100 \mathrm{mg} / \mathrm{Kg})$ injected intraperitoneally and a tracheostomy was made. A short arterial catheter was placed in the abdominal aorta through the right iliac artery for monitoring arterial pressure and for drawing blood samples for measurement of arterial $\mathrm{P}_{\mathrm{CO}_{2}}, \mathrm{P}_{\mathrm{O}_{2}}, \mathrm{pH}$ and arterial concentration of ${ }^{14} \mathrm{C}$. Two venous catheters were passed transfemorally into the bilateral iliac veins, the one for the injection of drugs and antipyrine $-{ }^{14} \mathrm{C}$, the other for an arteriovenous shunt. Each rat was paralyzed with a minimal dose of decamethonium and ventilated mechanically to maintain arterial $\mathrm{P}_{\mathrm{CO}_{2}}$ and $\mathrm{P}_{\mathrm{O}_{2}}$ relatively constant $\left(\mathrm{PaCO}_{2} 30-39 \mathrm{mmHg}, \mathrm{Pa}_{2}>100 \mathrm{mmHg}\right)$.

In 6 NTR and 7 SHR (experimental group), bilateral common carotid arteries were doubly ligated after careful dissection of cervical vago-sympathetic trunks. Approximately 2 hours after the carotid ligation, when the desired level of $\mathrm{PaCO}_{2}$ was reached, regional CBF was measured by the autoradiographic method (Reivich M et al: J Appl Physiol 27: 296, 1969) using $30 \mu \mathrm{Ci}$ of antipyrine- ${ }^{-14} \mathrm{C}$. Measurements of $\mathrm{rCBF}$ were made from various sites of each hemisphere and from the brain stem and the cerebellum.

In the remaining 7 NTR and 8 SHR (control group), a surgical preparation was performed in a similar manner as the experimental group except for

From the Second Department of Internal Medicine, Faculty of Medicine, Kyushu University, Fukuoka. 
the carotid ligation. The control values of rGBF were determined from the multiple sites.

The results obtained from the present study were as follows;

Mean regional blood flow values in the various sites of the brain in NTR and SHR of the control and experimental group were summarized in Table.

Table

\begin{tabular}{|c|c|c|c|c|c|c|}
\hline & & & \multicolumn{2}{|c|}{ Control Group } & \multicolumn{2}{|c|}{ Experimental Group } \\
\hline & & & NTR & SHR & NTR & SHR \\
\hline \multirow{6}{*}{ 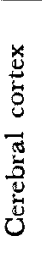 } & \multirow{3}{*}{ 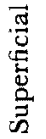 } & Frontal & $0.87 \pm 0.09^{*}$ & $0.85 \pm 0.10$ & $0.68 \pm 0.19$ & $0.17 \pm 0.21$ \\
\hline & & Parietotemporal & $1.07 \pm 0.07$ & $1.09 \pm 0.11$ & $0.73 \pm 0.19$ & $0.19 \pm 0.22$ \\
\hline & & Occipital & $1.05 \pm 0.13$ & $1.02 \pm 0.12$ & $0.71 \pm 0.20$ & $0.14 \pm 0.20$ \\
\hline & \multirow{3}{*}{ 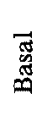 } & Frontal & $0.71 \pm 0.12$ & $0.81 \pm 0.22$ & $0.64 \pm 0.17$ & $0.20 \pm 0.18$ \\
\hline & & Parietotemporal & $0.89 \pm 0.07$ & $0.87 \pm 0.11$ & $0.64 \pm 0.18$ & $0.20 \pm 0.16$ \\
\hline & & Occipital & $0.86 \pm 0.15$ & $0.74 \pm 0.15$ & $0.55 \pm 0.17$ & $0.15 \pm 0.15$ \\
\hline \multicolumn{3}{|c|}{ Caudate nucleus } & $0.77 \pm 0.16$ & $0.79 \pm 0.19$ & $0.78 \pm 0.30$ & $0.19 \pm 0.19$ \\
\hline \multicolumn{3}{|c|}{ Putamen } & $1.05 \pm 0.11$ & $1.09 \pm 0.12$ & $0.75 \pm 0.21$ & $0.27 \pm 0.35$ \\
\hline \multicolumn{3}{|c|}{ Thalamus } & $1.35 \pm 0.15$ & $1.19 \pm 0.07$ & $0.91 \pm 0.12$ & $0.80 \pm 0.35$ \\
\hline \multicolumn{3}{|c|}{ Hippocampus } & $0.98 \pm 0.04$ & $0.85 \pm 0.10$ & $0.63 \pm 0.12$ & $0.21 \pm 0.16$ \\
\hline \multicolumn{3}{|c|}{ Brain stem } & $1.05 \pm 0.07$ & $0.98 \pm 0.13$ & $0.95 \pm 0.14$ & $0.83 \pm 0.23$ \\
\hline \multicolumn{3}{|c|}{ Gerebellar hemisphere } & $1.38 \pm 0.12$ & $1.25 \pm 0.15$ & $0.98 \pm 0.13$ & $0.96 \pm 0.31$ \\
\hline
\end{tabular}

* $\mathrm{rCBF}: \mathrm{m} / \mathrm{Gm} / \mathrm{min}, \quad$ Mean \pm S.D.

1) The blood flow values in the inferior surface of the cortex (basal cortex) were lower than those in the cortex of the convexity (superficial cortex) in both NTR and SHR. Similarly, blood flow values in the frontal cortex were lower than those in the parieto-temporal and occipital cortex in both NTR and SHR.

2) There was no significant difference in blood flow distribution of cerebral cortex between NTR and SHR. The mean rCBF in the thalamus, however, was higher in NTR than in SHR; this difference was statistically significant.

3) The ligation of bilateral carotid arteries produced a significant decrease of rCBF in both NTR and SHR, although the magnitude of decrease in $\mathrm{CBF}$ varied largely from animal to animal. However, the extent of ischemia appeared to be larger and the degree of ischemia more severe in SHR than in NTR. In NTR, the mean rCBF obtained from the various sites of the cerebral cortex and the subcortical gray matter ranged from 64 to $100 \%$ of the control group following the bilateral carotid ligation. In contrast, the mean $\mathrm{rCBF}$ in SHR ranged from 14 to $25 \%$ in the cerebral cortex and from 24 to 
$67 \%$ in the subcortical gray matter.

4) The rCBF in the brain stem and the cerebellum tended to decrease after the carotid ligation but the difference did not reach the statistically significant level. The rCBF values in the posterior circulation roughly correlated with those in the anterior circulation in NTR, but not in SHR.

It was reported by Eklöf and Siesjö (Acta Physiol Scand 87:69, 1973) that bilateral carotid ligation reduced $\mathrm{CBF}$ to about $50 \%$ of control values in NTR. In SHR, however, neither the magnitude of decrease in CBF following bilateral carotid ligation, nor absolute values of $\mathrm{rCBF}$ were reported previously.

In animals without bilateral carotid ligation, there was no significant difference of rCBF between NTR and SHR, despite the difference in the level of systemic blood pressure. Following the bilateral carotid ligation, the magnitude of decrease in $\mathrm{rCBF}$ of the anterior circulation was much larger in SHR than in NTR, which was in accord with the previous observation. 